\title{
Managing the operations of the TSU 2-m Automatic Spectroscopic Telescope
}

\author{
J.A. EATON and M.H. WiLliamSON \\ Center of Excellence in Information Systems, Tennessee State University, \\ 330 Tenth Avenue North, TSU Box 139, Nashville, TN 37203, USA
}

Received 12 July 2004; accepted 13 August 2004: published online 31 October 2004

\begin{abstract}
Tennessee State University has built and is now operating a completely automatic 2-m telescope for highdispersion spectroscopy. This system consists of an alt-azimuth $\mathrm{f} / 8$ Cassegrain telescope, housed in an enclosure with a roll-off roof, coupled to a white-pupil echelle spectrograph through a fiber-optic cable. We discuss managing this facility over the Internet with emphasis on 1) the configuration of the control system, 2) logging, 3) troubleshooting and quality control, 4) updating the target lists, and 5) archiving and handling the data.
\end{abstract}

Key words: telescopes - methods: data analysis - techniques: spectroscopic

(C) 2004 WILEY-VCH Verlag GmbH \& Co. KGaA, Weinheim

\section{Introduction}

Tennessee State University, which has been operating robotic telescopes for the past fifteen years, has built and since June, 2003, has been operating a 2-m telescope for high-dispersion spectroscopy (see http: / / astro.tsuniv. edu). Fig. 1 shows this Automatic Spectroscopic Telescope (AST). It is a classical Cassegrain with an f/1.5 primary and an aluminum secondary, giving an overall f/8 optical system. Because we designed the AST specifically for high-dispersion spectroscopy of stars, we placed the focus in front of the primary mirror, to reduce size of the secondary mirror, and supported the secondary with a quadrupod structure, to reduce interference between the telescope tube and the enclosure. The telescope acquires stars with a small video acquisition/guide camera looking at the telescope focus through a 45-degree pickoff mirror and sends light to the spectrograph via a 200-micron fiber-optic cable projecting through a hole in this mirror. The telescope guides on light spilling over the edge of the fiber optic cable (Libbrecht \& Peri 1995), with the position updated roughly every 2-5 seconds. For wavelength calibration/flat-fielding, we focus light at $\mathrm{f} / 8$ onto the fiber to the spectrograph which we bring into the guiding head from an external calibration bench through a 600-micron fiber.

The spectrograph itself is located in a separate enclosure, a well-insulated shipping container just south of the telescope enclosure. A second shipping container serves as a

Correspondence to: eaton@donne.tsuniv.edu

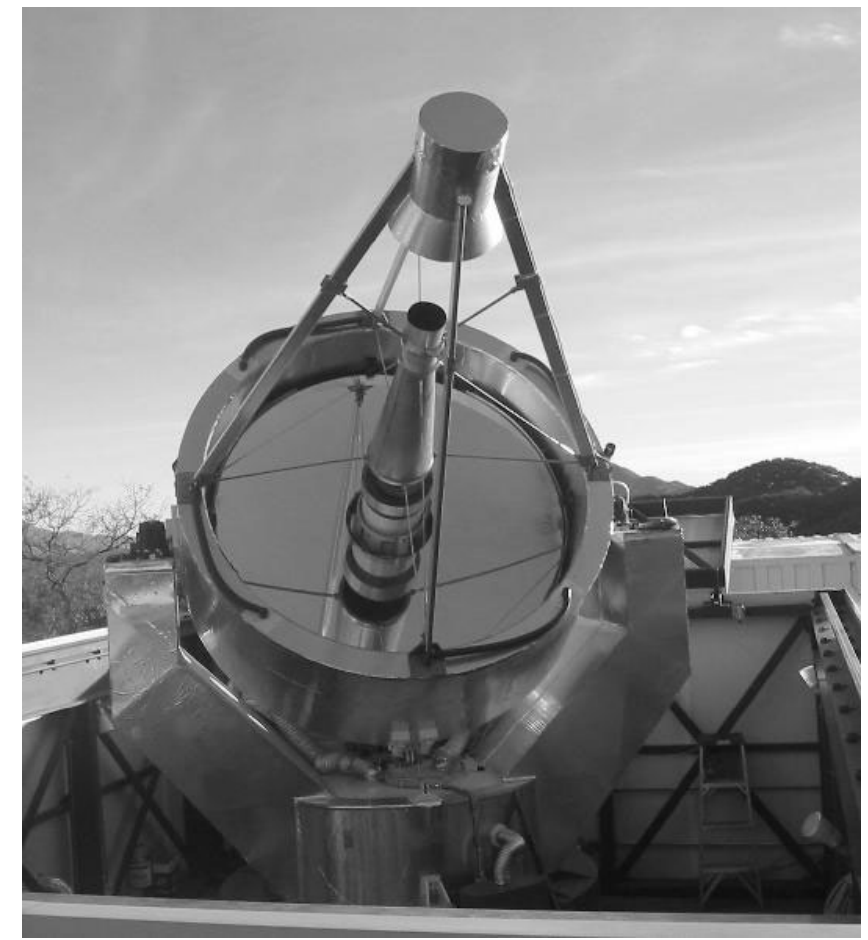

Fig. 1. The TSU 2-m AST in its observatory.

control room for the observatory. The spectrograph is a crossdispersed echelle of rather conventional white-pupil design 


\section{Network Access for TSU 2-m Telescope}

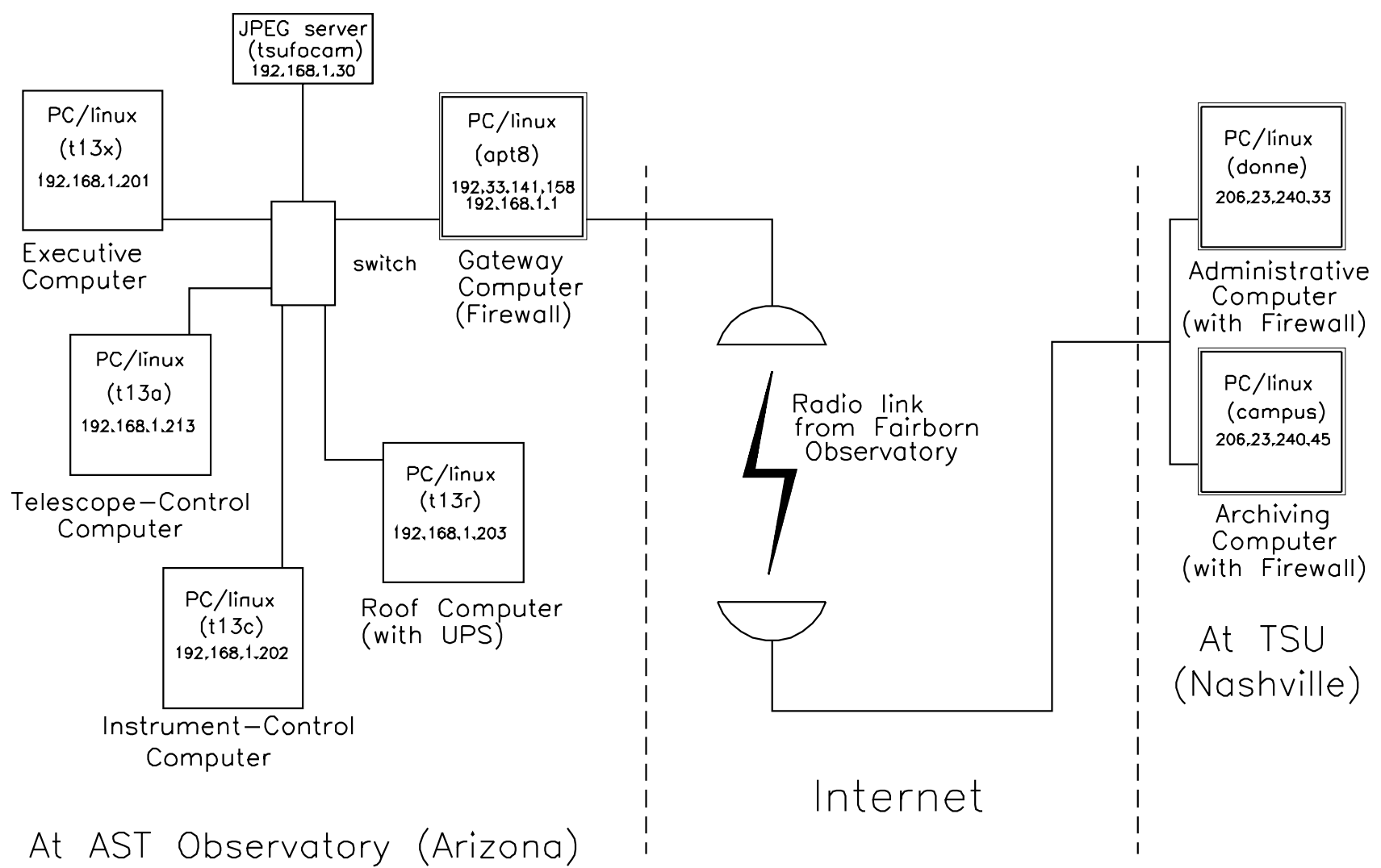

Fig. 2. A schematic view of the computers used to control and communicate with the TSU Automatic Spectroscopic Telescope.

(see Pilachowski et al. 1995) based on comercially available gratings, with a sophisticated camera designed by Harland Epps. We record the spectra with a SITe ST-002A CCD, controlled with an electronics package from Astronomical Research Cameras, Inc. We use the spectrograph in two wavelength ranges, 4900-7100 $\AA$ for the red and 3700-5700 $\AA$ for the blue. The standard resolution (approx. $\mathrm{R}=30,000$ ) is obtained by feeding the spectrograph with a bare 200-micron fiber, and a $\mathrm{R}=70,000$ mode, by using a 75-micron slit in front of a second fiber.

We operate the observatory with a control system running as background processes (daemons) on four computers networked behind a firewall. These four run 1) an executive process, 2) motion control, acquisition, and tracking of the telescope, 3) control of the spectrograph, and 4) motions of the telescope enclosure, respectively. They communicate either by exchanging UDP packets or by writing files to one another's discs. The control system incorporates many provisions to keep it from hanging up during the night and to make it restart gracefully when the computers reboot. Most of this software was written specifically for the AST at Tennessee State University by Williamson (Eaton \& Williamson 2004).

Nightly operations are started by cron jobs on the various computers, which either start the daemons at a particular time $(\mathrm{t} 13 \mathrm{x}, \mathrm{t} 13 \mathrm{c}$, and $\mathrm{t} 13 \mathrm{a})$ or make sure they are still running (t13r). The executive computer opens the observatory at the appropriate time $\left(\mathrm{ZD}_{\odot}=80^{\circ}\right)$ by sending a command to $\mathrm{t} 13 \mathrm{r}$, commands the spectrograph computer to make its calibration observations, runs through a list of stars, selecting observations to make throughout the night on the basis of ATIS-like priorities (see Henry 1996), keeps track of the weather, arranges to get calibration images at the end of the night, then shuts the observatory. The cron job on t13c makes sure the spectrograph control is running and initiates the data reduction at the end of the night.

We have discussed the control system more extensively at an SPIE conference in Glasgow (Eaton \& Williamson 2004). Here we should like to concentrate on the management and data-handling aspects of the observatory.

\section{Running the observatory}

Our 2-m telescope is located at Fairborn Observatory in southern Arizona, 1700 miles from Nashville, Tennessee, so we do not have the luxury of driving out to the site whenever we feel like it. So we have devised ways of managing the telescope remotely through the Internet. These mostly involve efficient use of logfiles and tools for file copying. For maintenance, we rely on Lou Boyd, Director of Fairborn Observatory, who has excellent technical skills for analyzing and fixing electronic, programming, and mechanical problems.

Fig. 2 shows the configuration of the computer connection between TSU and the 2-m AST. We use Fairborn Observatory's radio link to the outside world, roughly equivalent to 
a T1 line, to connect the telescope to the Internet. The control system is protected by a firewall that limits access to only four computers at TSU. These computers are themselves protected by firewalls that restrict the services they provide. We also try to limit communication with the observatory to the secure-shell protocol ( $\mathrm{ssh}$ and $\mathrm{scp}$ ) to keep from sending naked passwords over the Internet. Of course, this level of restriction means it is impossible to administer the AST from a random site.

\subsection{Directory structure and file copying}

To keep track of the many files for a given date, and to distinguish between files with the same name for different nights, we write all the files for a given night to a subdirectory for that night's Modified Julian Date number, e.g., /archive/mjd53001 or /home/ast/logfiles/mjd53001. Furthermore, we identify the reduced spectra we are putting into our database with the name of the night, as in AST53001-017.fits where "017" is a running image number. This type of identification follows the practices of Fairborn Observatory, and it avoids typing errors in administering the telescope by using the name of the subdirectory to set up all the file names.

To keep track of the information that must be fetched back from the observatory, we have automated the copying task. Information for a typical night consists of a large number of files with names like those in Fig. 3. These form two groups: 1) logfiles assembled on the gateway computer, apt8, at the end of the night by a cron job, and 2) files from the data reduction on the spectrograph computer, $\mathrm{t} 13 \mathrm{c}$. We fetch these groups of files back to Nashville by running programs in the subdirectory for the night, mjd53001 in our example. The program for copying logfiles identifies the night from the subdirectory name, sets up a secure-copy command to get the files, and executes it. We usually set up $\mathrm{X}$-windows with an agent (ssh-agent \& ssh-add) to keep from having to type a passphrase. For the reduced spectra, we run another copying program in the subdirectory for the data. It similarly sets up and runs a number of secure-copy commands to retrieve the reduced spectra and quality-control data for the reduction. This is a bit more complicated than copying the logfiles, since we must tunnel through the firewall to get to the spectrograph computer. Naturally we use a separate program to establish the tunnel.

\subsection{Disaster control}

Some things need to be checked every morning to make sure the telescope is not in danger. Mostly this means seeing that it has stowed and that the enclosure has closed automatically. This is almost always the case, for the roof controls designed and built by Lou Boyd (with the control program written by Don Epand, modified extensively by Boyd, and maintained by TSU) have worked very reliably. Nevertheless, several logical errors have cropped up in the roof-control program, and the roof has failed to shut when expected on a couple of nights with high humidity. These are only minor inconveniences. What we are concerned with are failures of the azimuth bearing in the telescope or problems with the drives

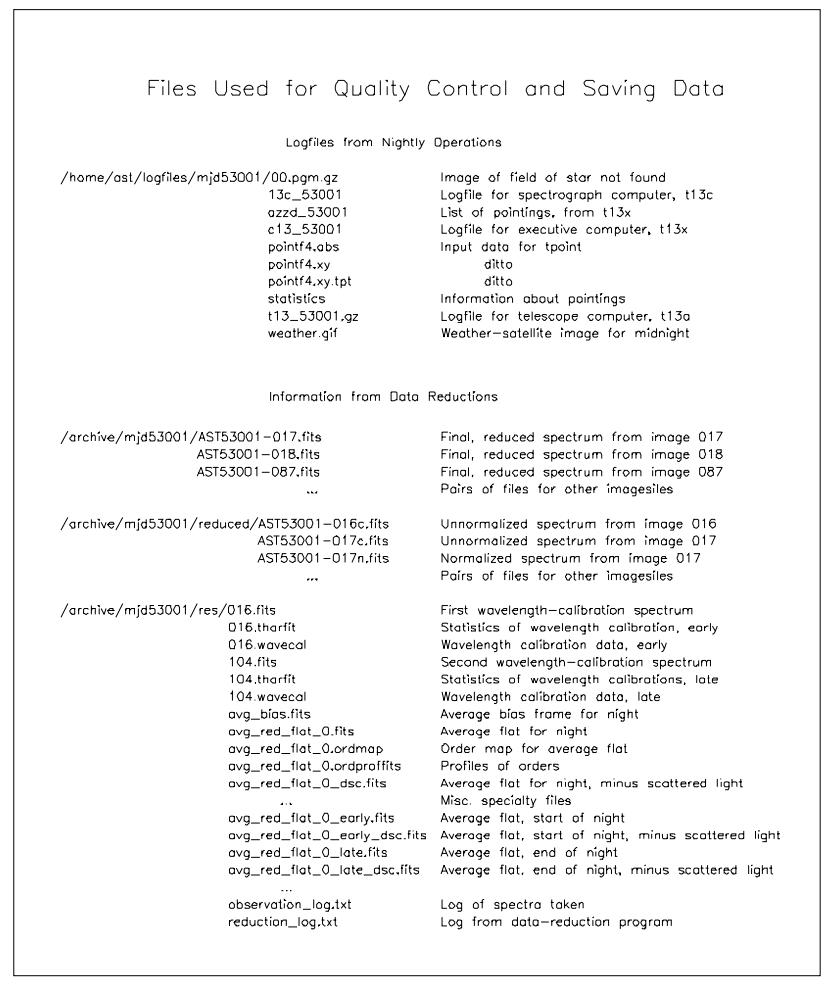

Fig. 3. Sample of files generated by the AST.

that would leave the telescope unstowed and possibly focusing sunlight on the enclosure walls, or in a failure of the roof drive that would leave the telescope exposed to bad weather for long periods.

To make sure the observatory shuts down properly, we use the Internet to monitor it in two ways. First, we send an E-mail back to Nashville with a copy of the log from the roof computer giving times for all the commands to the roofcontrol process and telling the status of the enclosure at those times, along with any error messages associated with these moves. Second, we have a video camera with a fisheye lens, connected to a JPEG server, by which we fetch a picture of the telescope every minute for a private web server. This lets us see directly where the telescope is pointed and whether the enclosure is closed. Both of these techniques have been quite useful for assessing problems with the enclosure drives. The video camera is especially useful for testing changes to the roof-control program we have made over the Internet.

\subsection{Keeping track of operations}

An instrument as complicated as the AST has numerous ways of failing, so we keep track of its operations through extensive logfiles written by the various control computers. The executive computer keeps track of when the control system opens and shuts the enclosure, which stars it orders the telescope to observe, whether the telescope acquires them, and when the tracking fails. The telescope computer logs the position of the telecope, pointing errors, and the demand on the servos every 3 seconds. It also logs once a minute the status of the oil pump and enclosure as well as the temperature and humid- 


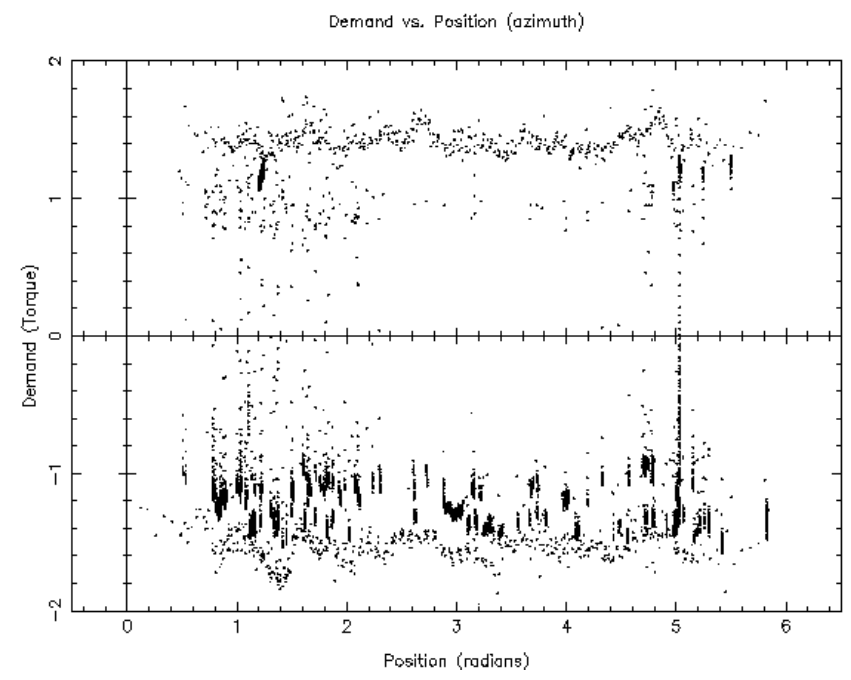

Fig. 4. The variation of demand (volts on 9-volt scale) on the servos for azimuth. Negative demand represents normal tracking (east to west in the south). Points are concentrated where the telescope was tracking, and the two outlying continuous bands correspond to slewing. You may notice one point to the east (1.4 radians) with somewhat higher friction.

ity in the observatory. Finally, it logs errors about such things as finding and tracking stars and slipping drives. The spectrograph computer logs temperatures of the CCD, telescope control room, and spectrograph enclosure every minute. It also records times at which it starts observations, when it reads out the $\mathrm{CCD}$, and when it records exposures in image files. Other information logged by the control programs includes errors of pointing while acquiring stars, which we could use for improving the telescope mount model, and images of starfields when the telescope did not find a star, which are useful for assessing the weather and problems with telescope focus. We also record an infrared image from a weather satellite at midnight, which gives a reasonable picture of the transparency for the night.

To make sense of all this information in the logfiles, we have written a program qc_t 13 to parse the logfiles, digest the data, and present the information in an understandable form-either as graphs, numerical assessments, or error messages, with appropriate pauses to look at the information presented.

\subsubsection{Information from the executive computer}

Information logged by the executive computer includes statistics about how many stars the telescope acquired, could not acquire, or lost while tracking. It gives us a very good indication of the quality of the night, how well the telescope is operating, how well the telescope is loaded by the starlists, and whether there are errors in the starlist. This information is summarized numerically on the computer screen and logged for further analysis. Plots used here are an infrared image from a weather satellite for midnight, which shows whether the night was cloudy, and plots of pointings in both altitudeazimuth and RA-dec, which indicate how well the observing program loads the telescope and where in the sky it is missing stars.

\subsubsection{Information from the telescope computer}

Most of the critical information comes from the telescope computer. It keeps track of how the telescope is acquiring and tracking stars. It also logs information about temperature of the environment and status of the oil pump and and positions of the moving parts of the telescope enclosure. Of particular interest here are plots of 1) how the temperature and humidity changed through the night, 2) how demand on the servos changes, which tests for mechanical problems in the drives such as sticking oil pads, and 3) variation of the guiding errors, which tell how well the telescope was tracking and also how good the night was. Fig. 4 shows an example of the record for servo demand.

\subsubsection{Information from the spectrograph computer}

The spectrograph computer logs information about performance of the CCD controller and settings of the spectrograph. It also keeps track of the temperature of the CCD and the spectrograph enclosure. Information parsed by qc_t 13 includes these temperatures as well as records of what images the spectrograph obtained and their quality. We list on the screen, for instance, names of the FITS image files saved, the objects observed, and the standard deviation of data in the image. This standard deviation is very useful in assessing the quality of the spectra of stars and for making sure the calibration lamp has not burned out.

\subsection{Real-time monitoring and programming}

The control system for this telescope is still somewhat of a work in progress in that we cannot presume to think of all the ways we might need to schedule and use the telescope $a$ priori. Also, bugs and logical inconsistencies in a program as complicated as a telescope control system are inevitable. So we need to be able to change the control software and test it remotely as needed by circumstances.

It is possible actually to run the telescope manually for testing, although this is easiest to do at the observatory. We can run the control processes for the telescope in the foreground with the logged data printed to the computer screen, although the executive process requires a separate program tel_control_front to enter manual commands into it.

Alternatively, we can monitor the telescope's progress by listing the contents of logfiles as they are being generated to determine how well the control system is performing some task. This is easy and convenient to do over the Internet.

\section{Reducing and keeping track of the spectra}

The spectrograph computer, $\mathrm{t} 13 \mathrm{c}$, uses a cron job to start the spectrograph control daemon, copy the collected images to a logging directory at the end of the night, and reduce the data collected. We reduce the data with a program written by 
Jeff Hall of Lowell Observatory; it is a generalization of the program he wrote to reduce spectra from the Lowell solarstellar spectrograph (see Hall et al. 1994). Reduced spectra are stored in FITS files consisting of an array of wavelengths and intensities for a certain number of 2048-pixel orders of the echelle spectra. These have to be archived and knitted together to get rid of the order structure with its overlapping but inconsistent wavelengths. To do this, we 1) copy the data back from the observatory as explained above, 2) run a quality-control program to look at the quality of the spectra, 3 ) renormalize intensities in the various orders, weight the intensities roughly to reflect the echelle blaze function, and map them onto a common wavelength scale, and 4) make a record of each final reduced spectrum in a database. Each fully reduced spectrum requires about 240 kilobytes of storage, and the three forms of reduced spectra we are saving require of the order of $1 \mathrm{MB}$.

\section{Scheduling and updating the observing program}

We decided not to use ATIS, the Automatic Telescope Instruction Set (Boyd \& Epand 2000), to specify the observations because it seemed to add an extra, unnecessary level of complexity to our operations. Instead, we specify the observations for a given night as a table of stars with general priorities of observation (star.list), corresponding to the frequency we would like them observed. An algorithm written into the executive program calculates instantaneous priorities from these general priorities with a set of ATIS-like rules. It also gives us the ability to specify observation of a star at a specific time regardless of its general priority. The control system sets up the spectrograph at the beginning of the night according to the type of observation specified in the star. 1 ist file for the night. It then takes bias and calibration spectra and waits for time to start observing stars $\left(\mathrm{ZD}_{\odot}\right.$ $=100^{\circ}$ ). At the end of the night, it takes more bias and calibration spectra, stows the telescope, shuts the observatory, and reduces the data.
Because of the way the control system selects stars, it would observe essentially the same stars on consecutive nights, changing the mix slowly over the year as different parts of the sky became exposed. This is fine if all the stars should need observing at roughly the same frequency or if the telescope is not overloaded, and it is actually pretty much what our photometric telescopes do. However, the overloaded, inhomogeneous program for this spectroscopic telescope must sometimes be adjusted on a daily basis to take into account observations made on previous nights. We have written a scheduling program, freq_t 13 , to read a master stars list, look back at summaries of observations taken of those stars over a specified number of nights, and adjust the general priorities to favor stars not observed at a frequency proportional to their base priorities. It then writes a new stars file, which we can copy to the executive computer, $\mathrm{t} 13 \mathrm{x}$, through another tunnel through the firewall. This procedure has been especially useful for managing monitoring programs with long exposures for which only a relatively small fraction of the stars get observed on a given night.

Acknowledgements. We are grateful to Lou Boyd for help with and advice on many of the computer applications we have discussed. The following TSU students contributed to writing and checking control/processing software in a material way: Melvin Poplar, Shawn Vaughns, Allen Keel, and Sean Williams. We should also like to acknowledge financial support of the AST program from NASA, NSF, and the State of Tennessee.

\section{References}

Boyd, L.J., Epand, D.: 2000, http://www.fairobs.org/atis2000.html Eaton, J.A., Williamson, M.H.: 2004, SPIE 5496, in press

Hall, J.C., Fulton, E.E., Huenemoerder, D.P., Welty, A.D., Neff, J.E.: 1994, PASP 106, 315

Henry, G.W.: 1996, ASP Conf. Ser. 87, 145

Libbrecht, K.G., Peri, M.L.: 1995, PASP 107, 62

Pilachowski, C., Dekker, H., Hinkle, K., Tull, R., Vogt, S., Walker, D.D., Diego, F., Angel, R.: 1995, PASP 107, 983 